Fetal Diagnosis and Therapy
Fetal Diagn Ther 2019;46:159-165

DOI: $10.1159 / 000494617$
Received: August 24, 2018

Accepted after revision: October 17, 2018

Published online: November 21, 2018

\title{
Refining the Prediction and Prevention of Emergency Operative Deliveries with the Fetal Reserve Index
}

\author{
David W. Britt ${ }^{a}$ Mark I. Evans ${ }^{a}$ b Barry S. Schifrin ${ }^{a}$ Robert D. Eden ${ }^{a}$ \\ ${ }^{a}$ Fetal Medicine Foundation of America, New York, NY, USA; ${ }^{b}$ Comprehensive Genetics, PLLC/Department of \\ Obstetrics and Gynecology, Mt. Sinai School of Medicine, New York, NY, USA
}

\section{Keywords}

Electronic fetal monitoring · Fetal Reserve Index · Neonatal encephalopathy · Cerebral palsy · ACOG monitoring

classification system $\cdot$ Intrauterine resuscitation

\section{Abstract}

Electronic fetal monitoring (EFM) is a poor predictor of outcomes attributable to delivery problems. Contextualizing EFM by adding maternal, obstetrical, and fetal risk-related information to create an index called the Fetal Reserve Index (FRI) improves the predictive capacity and facilitates the timing of interventions. Here, we test critical assumptions of FRI as a clinical tool. Our conceptualization implies that the earlier one reaches the red zone ( $F R I \leq 25)$ and the longer one spends in the red zone, the greater the likelihood of emergency operative deliveries (EOD). Methods: We analyzed 1,402 patients using logistic regression predicting the probability of EOD and employed qualitative methodology techniques to refine predictive capabilities. Results: Reaching the red zone early and staying there $>1 \mathrm{~h}$ increases the probability of EOD. When these risk factors are paired with intrauterine resuscitation (IR) in Stage 1, the reduction of EOD is substantial. Conclusion: FRI is a capable predictor of EOD because it accurately identifies the level of malleable risk. FRI analysis increases the risk of using IR in Stage 1. Matching risk and resources dramatically reduces the chances of EOD. Earlier IR improves the outcomes if the calculated risk is high.

(c) 2018 S. Karger AG, Basel

\section{Introduction}

A broad array of research has shown that electronic fetal monitoring (EFM) in actual practice has a low ceiling of effectiveness. The poor metrics have an impact on both the prediction and prevention of cerebral palsy and other deleterious outcomes attributable, at least in part, to problems during labor and delivery [1-8]. Severe criticism has also come from those who believe that EFM fails on medical, legal, and ethical grounds and should be limited or abandoned $[9,10]$.

We have developed the Fetal Reserve Index (FRI), which combines 5 quantifiable components of the fetal heart tracing (rate, variability, accelerations, decelerations, and the presence of increased uterine activity [IUA]) but then contextualizes EFM by adding 3 clinical variables: (1) maternal, (2) obstetrical, and (3) fetal risk-

\section{KARGER}

(c) 2018 S. Karger AG, Basel

E-Mail karger@karger.com

www.karger.com/fdt
Mark I. Evans, MD

Comprehensive Genetics, PLLC/Department of Obstetrics and Gynecology Mt. Sinai School of Medicine

131 E 65th Street, New York, NY 10065 (USA)

E-Mail evans@compregen.com 
related information. Collectively, these create an 8-point index of risk, the FRI [11-13].

Our previous studies have shown that the FRI significantly improves the predictive capacity and allows the identification of pregnancies at an increased risk of adverse outcome far earlier in the sequence of deterioration than traditional methods, thus affording time and opportunity to prevent both harm and the need for emergency operative deliveries (EOD) [11-13].

In this study, we incorporate sophisticated statistical approaches more commonly used in the social science literature, which allow us to gain a deeper understanding of the interactions of different clinical factors. We can then build on our own prior research to evaluate some of the critical assumptions underlying the FRI as a clinical tool and its ability to marshal fetal therapeutic activities (intrauterine resuscitation [IR]).

We have hypothesized that the earlier one reaches the screen positive state (called the "red zone", which is an FRI score of $\leq 25 \%$ ) and the longer the duration one spends in the red zone, the greater the likelihood of both fetal harm and the need for EOD - a risk factor in itself for cerebral palsy and other adverse outcomes. In this study, we test these assumptions on an expanded study group to determine if certain aspects of the FRI are more informative about outcomes than others. Specifically, we developed statistical models to examine how the red-zone risk increases the chances of EOD and what happens to the chances of EOD when these risks are paired with IR during the 1st stage of labor.

\section{Methods and Materials}

\section{Population}

Our current study set consists of 1,402 control patients (i.e., all with normal outcomes) and expands upon earlier studies in which we consider risks in conjunction with interventions. All study deliveries occurred between 2013 and 2016. They were at term $(\geq 37$ weeks) with singleton pregnancies in vertex presentation who, irrespective of any maternal complications, presented for a trial of labor with Category I, normal fetal heart rate (FHR) patterns, and no evidence of prior neurological injury or anomaly (Table 1).

Variables and Statistical Analysis

The FRI evaluates 3 broad, clinical components (maternal, fetal, and obstetrical) and 5 EFM components (FHR baseline, accelerations, decelerations, variability, and uterine activity). Except for an enhanced definition of IUA, we used standard American College of Obstetricians and Gynecologists (ACOG) definitions. The EFM parameters and broadly accepted risk factors are treated as individual parameters and have been defined extensively and vetted elsewhere. Briefly, medical factors include hypertension, diabetes, maternal age, $\mathrm{BMI}>40$, and nulliparity, as well as chronic
Table 1. Descriptive sample characteristics

\begin{tabular}{|c|c|c|}
\hline & Mean (SD) & $n(\%)$ \\
\hline Age, years & $27.86(5.88)$ & \\
\hline \multicolumn{3}{|l|}{ Parity } \\
\hline $\mathrm{P}$ & & $636(43.5)$ \\
\hline M & & $826(56.5)$ \\
\hline Gestational age, weeks & $38.66(1.83)$ & \\
\hline Birth weight, $g$ & $3,247.98(553.16)$ & \\
\hline \multicolumn{3}{|l|}{ Red1h+ } \\
\hline 0 & & $1,227(83.9)$ \\
\hline 1 & & $234(16.0)$ \\
\hline \multicolumn{3}{|l|}{ Worst1Red } \\
\hline 0 & & $1,217(83.2)$ \\
\hline 1 & & $245(16.8)$ \\
\hline \multicolumn{3}{|l|}{ Stage1_IR } \\
\hline 0 & & $1,097(75)$ \\
\hline 1 & & $365(25)$ \\
\hline \multicolumn{3}{|l|}{ EOD } \\
\hline 0 & & $1,220(83.4)$ \\
\hline 1 & & $242(16.6)$ \\
\hline
\end{tabular}

medical disorders (e.g., collagen, respiratory, or cardiac). Obstetrical variables are gestational age, need for induction of labor, use of oxytocin and/or prostaglandin, duration of labor (by stage), and type and urgency of delivery. Fetal factors are abnormal Doppler/ biophysical profile, evidence of growth or genetic disorders, meconium, etc.

These factors are combined to define an 8-parameter algorithm (Tables $1-3$ in Eden et al. $[11,12]$ ). If all 8 are normal: $8 / 8=100 \%$; $2 / 8=25 \%$, etc. EFM scores and IUA are dynamic and change numerically (with associated color zone) as a feature improves or deteriorates. Most clinical risk factors, however, are unidirectional (e.g., diabetes, ruptured membranes). FRI scores $>50 \%$ are considered green zone, between 50 and $26 \%$ yellow zone, and $\leq 25 \%$ red zone, which we interpret as the definition of screen positive and "at-risk status" and evoking concern demanding affirmative attention and written evaluation.

Using the FRI as the primary determinant of action would, of course, be abandoned with certain "sentinel" events - clinical maternal, fetal, or obstetrical events that represent sufficient emergency indications for intervention per se. These might include, among others, uterine rupture, umbilical cord prolapse, maternal hemorrhage, seizures, coronary compromise, and a persistent FHR bradycardia that persisted for $>10 \mathrm{~min}$, although no such examples were present in this series. EOD, as used in this paper, were reserved for emergency cesarean section, vacuum, or forceps deliveries that were only performed for acute fetal compromise (i.e., terminal bradycardia, repetitive late decelerations unresponsive to IR).

Likewise, we considered IR using standard protocols that have been used for the past 4 decades. Components may include: (1) cessation of pitocin administration; (2) cessation of maternal pushing efforts in the 2nd stage of labor; (3) use of tocolytic agents (i.e., terbutaline) in the presence of tetanic uterine contractions or polysystole); (4) maternal positional change to alleviate significant 
FHR changes (i.e., prolonged deceleration); (5) i.v. fluid infusion to expand the intravascular blood volume of the mother to alleviate hypotension; (6) oxygen administration via mask to increase the oxygen content of the maternal blood; and (7) pelvic examination to check for cervical dilation, station, and prolapsed umbilical cord.

The current analysis uses binary logistic regression to evaluate the relative influence of different risk and intervention factors on the risk of emergency births. Logistic regression is a form of regression analysis developed for use with categorical outcome variables. When the outcome is categorical, the linearity assumption of regression analysis is violated. Logistic regression gets around this by logarithmically transforming the regression equation. Hence, instead of predicting the value of an outcome variable from a set of predictor variables, logistic regression predicts the log odds of a categorical outcome variable given a set of known predictor variables and probabilities derived from $\operatorname{Exp}(\mathrm{B})$ using the formula

$$
\text { Probability }=\operatorname{Exp}(\mathrm{B}) / \operatorname{Exp}(\mathrm{B})+1 .(1)
$$

In our case, we are predicting the log odds of requiring an "emergency" birth (EOD) from a set of dichotomous predictor variables: 1 whether a patient has reached the FRI red zone during the 1st stage of labor (RedStage1, coded as 1 or 0 )

2 whether a patient who reached this risk level has remained in the red zone for at least $1 \mathrm{~h}(\operatorname{Red} 1 \mathrm{~h}+$, coded as 1 or 0$)$

3 whether timely efforts were made to moderate the risk with IR in the 1st stage of labor (Stage1_IR, coded as 1 or 0 ).

The contingent nature of the analysis is reflected in the introduction of two interaction effects, which pair risk (duration of time in red zone and the initiation of IR):

1 RedStage1 X Stage1_IR, coded as 1 when both occur and 0 otherwise, operationalizes whether there was a co-occurrence of high-risk red zone in the 1st stage of labor and the timely initiation of IR.

2 Red1h+ X Stage1_IR, coded as 1 when both occur and 0 otherwise, operationalizes whether there was co-occurrence of high risk represented by being in the red zone for at least $1 \mathrm{~h}$ and the initiation of IR in Stage 1.

This is not a pure academic exercise because in analyzing FRI as a screening tool, we are examining both factors that summarize risk in the context of an intervention that may serve to minimize those risks under certain conditions. Thus, our primary focus is the analysis of the contingent nature of reversible risk with IR.

Logistic regression facilitates the conceptual model type of analysis, but to improve the analysis, we display our expectations more graphically [14] using two conceptual models (Fig. 1, 2). Figure 1 displays our expectations regarding the impact of getting to the red zone in Stage 1 (Worst1Red) and staying in the red zone for at least $1 \mathrm{~h}(\mathrm{Red} 1 \mathrm{~h}+)$. Net of one another, we expect each of them to have a positive, strong impact (as indicated by the plus [ + ] sign) on the probability of EOD. Figure 2 enables us to examine more closely the implications of intervening with IR during the 1st stage of labor. The solid lines represent the continuing threat represented by prolonging the time in the red zone. The dashed lines represent interaction effects in which IR in the 1st stage is paired either with having reached the red zone during this time or by being in the red zone for $\geq 1 \mathrm{~h}$. Here, we expect that the red zone characteristics represent a continuing threat, but when they are paired with the use of IR in the 1st stage, the probability of EOD is greatly reduced (as indicated by the minus [-] sign).

Improving the Prediction of Emergency

Delivery with the Fetal Reserve Index

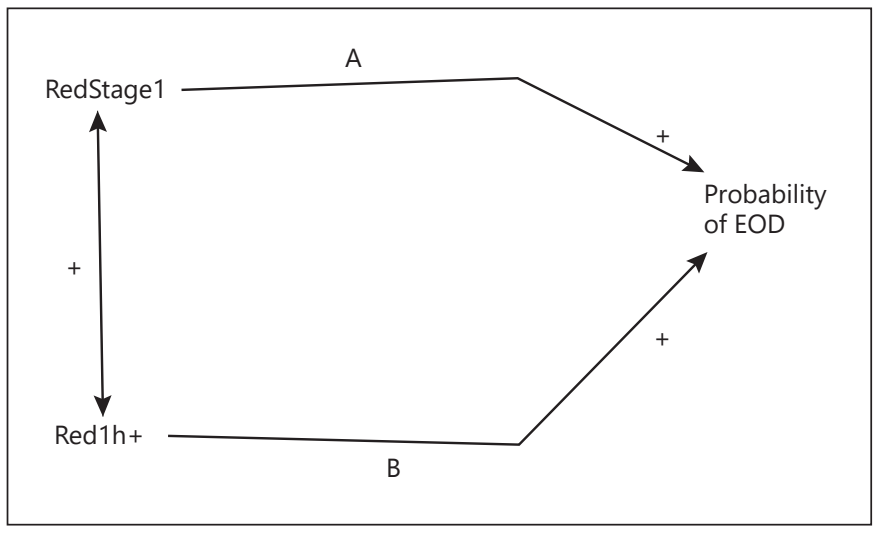

Fig. 1. The variables in this conceptual model are arranged in a recursive order, from most exogenous to outcome (in a rectangle for clarification), as per convention (Britt [14]). Double-headed arrows reflect a simple association. Single-headed arrows reflect the direction of influence. Upper-case letters A and B reflect alternate paths. Plus $(+)$ signs indicate that variables move in the same direction, so, for example, path B signifies that being in the red zone for $\geq 1$ h leads to a higher probability of EOD.

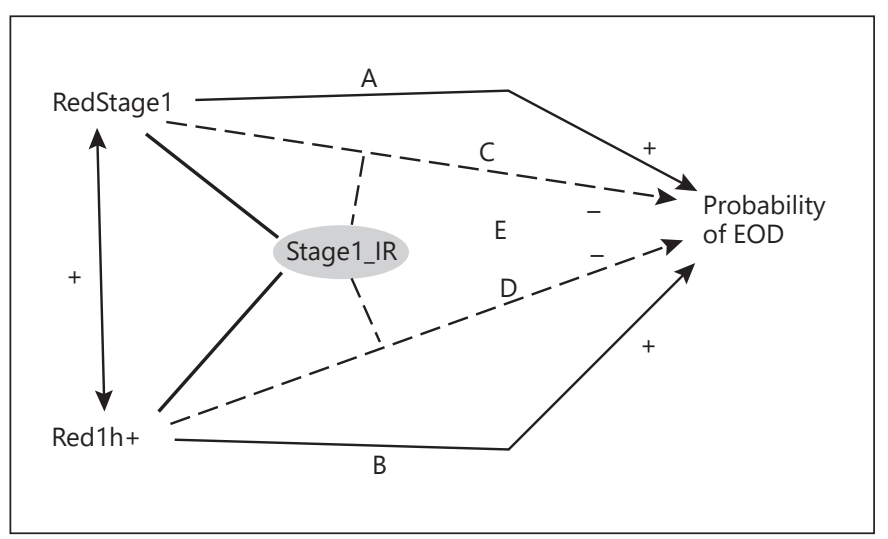

Fig. 2. Solid lines represent additive effects (A and B as before). Dashed lines represent interaction effects $(C$ and $D)$ : reaching the red zone by Stage 1 combined with the use of IR in Stage 1, and being in the red zone for $>1 \mathrm{~h}$ combined with the use of IR in Stage 1. Interaction effects for Worst1Red and Red $1 \mathrm{~h}+$, respectively. The absence of lines (E, for example) represents no additive effect. Plus $(+)$ signs indicate variables that move in the same direction. Minus signs (-) indicate variables that move in the opposite direction. Both interaction effects reduce the probability of EOD.

\section{Results}

About $16 \%$ of the patients were Red1h+, Worst1Red, and had EOD. However, 25\% had Stage1_IR (Table 1). Of the 1,402 patients in our sample, there were 186 patients 
Table 2. Model containing FRI red-zone variables

\begin{tabular}{lrllllllrr}
\hline & B & SE & Wald & df & Sig. & Exp(B) & Lower 95\% CI & Upper 95\% CI & Prob. \\
\hline Red1h+ & 1.672 & 0.220 & 57.77 & 1 & 0.000 & 5.321 & 3.458 & 8.184 \\
Worst1Red & 2.050 & 0.209 & 96.11 & 1 & 0.000 & 7.772 & 5.158 & 0.84 \\
Constant & -2.819 & 0.123 & 523.75 & 1 & 0.000 & 0.060 & & 0.89 & 11.710 \\
\hline
\end{tabular}

(about 13\%) who had emergency deliveries, and 1,216 who did not.

Empirically, confronting the implications of the FRI as a clinical tool for reducing the chances of EOD requires being able to jointly consider the influence of both risk and therapeutic interventions. Binary logistic regression permits the assessment of the relative impact of relevant variables and their interactions. These generate probabilities of EOD given different combinations of relevant variable percentages of variance explained and the improvement in predictive capacity of the model. Figures 1 and 2 thus diagrammatically summarize our expectations as conceptual models [14]. We have developed these conceptual models to demonstrate the complexity in predicting the interactions of multiple variables and their interaction effects.

\section{Model 1: Model Based on Red-Zone Risk Factors}

Table 2 presents the results of a logistic regression that includes only the constant term (which summarizes the probability of EOD when both red-zone risk factors are coded as "0") and the two red-zone risk factors (Fig. 1). The two important predictions here are that both Worst1 Red (line A) and Red1h+ (line B) are strong and positive, indicating that they increase the chances of EOD. At first glance, this model looks good. This model has a pseudo $\mathrm{R}^{2}$ (as it is not really variance explained, Nagelkerke's $\mathrm{R}^{2}$ ) of 0.34 and an $88.4 \%$ accuracy in predicting whether patients will have an EOD or not. Further, the highly significant coefficients remain significant even after imposing an additional constraint recommended for running logistic regressions on larger samples (Raftery [15] cited in Pampel [16]: $\mathrm{BIC}=\mathrm{Z}^{2}-\ln \mathrm{N}$, which simply states that the Wald statistic should exceed the log of the sample size). The log of a sample size of 1,401 is 7.25 , and as can be seen from Table 2, the Wald statistics for both Red $1 \mathrm{~h}+$ (57.66) and Worst1Red (60.71) are comfortably above the threshold of the log of the sample size.

However, there are some serious shortcomings in this model. One way of assessing this is comparing the baseline accuracy of the placement of true-positive and true- negative cases to the improvement in accuracy generated by the model. The baseline accuracy (based solely on the constant) is $86 \%$, attributable to the relative rarity of EOD cases in our sample. The accuracy from model 1 is only $88.4 \%$, a negligible improvement of $2.4 \%$. Secondly, the model does not fit the data very well. SPSS produces the Hosmer-Lemeshow test [17]. If a model does a good job of fitting the available data, then this statistic should not be significant. If it does a poor job, the statistic should be significant. For this model, the Hosmer-Lemeshow statistic is significant at the 0.000 level, indicating that the model is misspecified in that it has left relevant variables out of the analysis. Thus, we created a second model.

\section{Model 2: Model Based on Red-Zone Risk Factors and Stage1_IR}

This second model adds the primary intervention suggested for patients who reach the red zone, in vivo resuscitation in Stage 1 of the delivery. Technically, Stage1_IR is not a confounding factor since it comes between the onset of the separable dimensions of red-zone risk and outcomes, but neither is it an explanatory variable in a chain of causes. Rather, it is a clinical intervention prompted by the onset of the risk factors designed to reduce the risk and allow the delivery to proceed normally. Logistic regression gives us the opportunity to examine this process more closely and see more clearly the implications of properly timed intervention and nonintervention.

Models may have several functions [14], and net of their variance explained or accuracy, if they clarify important aspects of clinical processes, they may be useful. Model 2 is built upon Model 1. We continue to expect that, net of the other variables in the model, both Worst1 Red (line A) and Red $1 \mathrm{~h}+$ (line B) will increase the chances of EOD. At the same time, we expect that the pairing of Stage1_IR with each of these risk factors will attenuate the probability of EOD (as presented by the negative signs for both lines $\mathrm{C}$ and line D). Further, we expect that net of these variables, Stage1_IR will not have any sort of effect on the probability of EOD, represented in the model as the lack of a line $\mathrm{E}$. 
Table 3. Full model with FRI risk factors and Stage1_IR

\begin{tabular}{|c|c|c|c|c|c|c|c|c|c|}
\hline & B & SE & Wald & $\mathrm{df}$ & Sig. & $\operatorname{Exp}(B)$ & Lower 95\% CI & Upper 95\% CI & Prob. \\
\hline Red1h+ & 2.565 & 0.304 & 71.30 & 1 & 0.000 & 13.004 & 7.169 & 23.586 & 0.93 \\
\hline Worst1Red & 2.686 & 0.464 & 33.47 & 1 & 0.000 & 14.666 & 5.905 & 36.429 & 0.94 \\
\hline Stage1_IR & 0.688 & 0.295 & 5.45 & 1 & 0.020 & 1.990 & 1.117 & 3.547 & 0.67 \\
\hline Stage1_IR by Worst1Red & -0.773 & 0.563 & 1.89 & 1 & 0.170 & 0.462 & 0.153 & 1.391 & 0.32 \\
\hline Stage1_IR by Red1h+ & -1.726 & 0.423 & 16.63 & 1 & 0.000 & 0.178 & 0.078 & 0.408 & 0.15 \\
\hline Constant & -3.076 & 0.156 & 388.33 & 1 & 0.000 & 0.046 & & & 0.04 \\
\hline
\end{tabular}

Table 4. Collinearity diagnostics for Model $2^{\mathrm{a}}$

\begin{tabular}{lll}
\hline & Tolerance $^{\mathrm{b}}$ & VIF $^{\mathrm{c}}$ \\
\hline Worst1Red & 0.166 & 6.025 \\
Red1h+ & 0.387 & 2.584 \\
Stage1_IR & 0.605 & 1.654 \\
Worst1Red_by_Stage1_IR & 0.137 & 7.294 \\
Red1h+_by_Stage1_IR & 0.305 & 3.278 \\
\hline
\end{tabular}

${ }^{a}$ Collinearity statistics is not available for logistic regression in SPSS. As a substitute recommended by Field (2013), the model was re-run using OLS regression. ${ }^{b}$ Tolerance is the proportion of variance for each variable that is not attributable to the other independent variables. Values close to 1.0 are safe, but as values approach 0.0 , collinearity becomes more of a problem. ${ }^{c}$ Variance inflation factors (VIF) is the reciprocal of Tolerance and conveniently interpretable in terms of the amount of inflation in the associated standard errors. So, for example, Worst1Red's VIF of 6.025 means that its standard error is more than 6 times what one would expect if there were no correlation among the independent variables.

Table 3 presents the coefficient results and the probabilities of EOD with other variables controlled. The Hosmer-Lemeshow test $\left(\chi^{2}=1.21\right.$. significance at $\left.<0.546\right)$ is not significant (indicating a good fit of the model to the data), and the pseudo $\mathrm{R}^{2}=0.37$. The accuracy of the model remains at $88 \%$. Applying the BIC constraint that in a large sample, the Wald statistic $\left(z^{2}\right)$ should be greater than the log of the sample size (in this case, 7.25), two of the coefficients do not pass muster in this regard. Neither the impact of Stage1_IR with other variables held constant nor the interaction effect of Stage1_IR by Worst1Red satisfy this criterion.

However, let us first concentrate on those things that appear to be significant. With the influence of other factors held constant, both red-zone risk factors (Red1h+ and Worst1Red) are very strong compelling predictors; each is associated with a probability of EOD $>90 \%$. Fur- ther, the interaction of Red1h+ and Stage1_IR appears very important as well, reducing the expected probability of EOD from 0.93 to 0.15 .

Despite these promising results, there is a problem with collinearity as the red zone characteristics are highly intercorrelated. Using collinearity, both Worst1Red and the interaction of Stage1_IR and Worst1Red are suspect (Table 4 ) with tolerance values $<0.2$ (values $<0.2$ are considered a potential problem, and values $<0.1$ are considered a major problem) and variable inflation factors; values $>6$ are considered suspect and scores of 10 are considered severely suspect). Further, the average of variable inflation factor scores - which is directly interpretable as the multiple by which a standard error is inflated relative to what one would expect if there were no correlation among the independent variables - for all variables in the model is $>4$, and if the average is $>1$, collinearity is a suspect. In the case of the model represented in Table 3, the inflated standard errors may be masking the significance of an important effect - the potential capacity of pairing Stage1_IR with having reached the red zone by Stage 1, thereby reducing the risk down to $32 \%$.

\section{Discussion}

Our approach here represents an attempt to mobilize the experience and decision-making skills, resources as their need increases. More generally, our concept is to incorporate methods more commonly used in the social science literature to enhance our diagnostic capabilities beyond that traditionally used in the medical sphere. Not only does our approach call attention to a problem, with some but not complete specificity, it keeps track of how long that problem has been present. The latter is done in a rigorous fashion not typical of medical statistics. The prescription envisions not only corrective measures, which are the responsibility of the care givers even with 
minimal experience. Further, it prescribes timely consultation with the attending physician.

We anticipated that using IR without considering the level of risk would be akin to bringing the resources and knowledge provided by a subspecialist to bear on a routine case (of any type). This circumstance brings greater expertise and expense but likely offers little measurable improvement in the outcome [18]. The parallel is that both entail an understanding that risk and resources must be matched. Such balancing of risks and benefits by adopting new technologies and reconsideration of long held dogmas to allow earlier recognition of problems is being developed in other areas of care such as relying on screening versus diagnostic testing in prenatal diagnosis [19-21].

We have argued that there are several critical assumptions regarding the use of FRI as a clinical tool. If FRI is to be considered a genuine improvement over EFM by itself, the mechanics of using FRI must be put to the test. Two of these assumptions simply describe the deleterious effects of reaching the red zone early and/or staying there, unrecognized, for at least $1 \mathrm{~h}$. Without IR, the probability of EOD shifts from low 0.13 to over 0.80 in both of these situations. One of our hypotheses that must be proven with larger study samples is the effect of the FRI on initiating IR due to excessive uterine activity or worsening FHR patterns. In a previous report, we demonstrated the reduction of EOD with aggressive IR, which in part is due to the reduction in the number of contractions to $<5$ per 10 min - considerably lower than the ACOG definition of 6 contractions in $10 \mathrm{~min}$. We believe the reduction in time for the fetus to recover from cessation of oxygen passage to the uterus and the fetus during contractions is important. Our data strongly suggest that with the reduction of contractions and aggressive IR, the fetus is more likely to be able to tolerate the effects of second-stage labor, which often result in terminal bradycardia after excessive periods of decreased FHR variability, loss of accelerations, and late- or slow-return-to-baseline decelerations.

Importantly, however, we have shown that the contingent use of IR under these conditions profoundly lowers the chances of an EOD. Collinearity in the data precludes more confidence in this finding for Worst1Red because of an inflated standard error, and the same caveat applies to our tentative finding that simply using IR without reference to the level of risk identified by FRI does not result in such beneficial outcomes.

Strengths of our study include a large cohort of control laboring patients analyzed by our system demonstrating the ability of the FRI to predict impending compromise. Limitations include the need for computerization of our system (in progress), which will allow substantially larger data analysis in a rapid fashion. Once computerization is complete, the diagnoses of risk factors, etc. will come directly from the electronic medical record. For the hand calculated cases we have used to date, diagnoses were taken from the chart by a maternal fetal medicine sub-specialist reviewer. While there will always be the possibility for errors, there is no reason to believe they should be systematically biased in any particular direction. The analysis of much larger data sets will also mitigate the influence of the collinearity in our current data set, which is important because collinearity can complicate the interpretation of some of the effects we found.

Our analysis centers on broadening the FRI approach to the management of labor. The FRI focuses on the earlier identification of problems before they become serious rather than on the identification of problems once they have occurred. For 50 years, there has been widespread confusion in practice on the "front lines" of labor and delivery as to whether EFM is a screening or a diagnostic test [6]. The lack of rigor and subjectivity in the interpretation of EFM has led to widely divergent "expert opinions," which has directly filtered down into the medicolegal arena, and greater controversy.

Over the past several decades, there have been significant increases in the use of cesarean sections, many of them on an emergent basis, yet with little apparent improvement in the prevention of neonatal compromise and at an enormous increase in costs to the medical system. We believe our approach can lead to a better value proposition and greater standardization of screening and response to changes in labor with hopefully improved outcomes with less intervention and at lower costs.

\section{Statement of Ethics}

Demographic, EFM, and clinical maternal, obstetrical, fetal, and neonatal variables were deidentified for the analysis and as such qualified for exemption as evaluated by the Biomedical Research Association of NY IRB (No. 16-12-180-429).

\section{Disclosure Statement}

M.I.E. has obtained a US patent for the scoring system (US Patent $9,131,860$, with others pending). There was no external funding, and there are no conflicts of interest related to such. 


\section{References}

1 Cibils LA. Interpretation of intrapartum fetal heart rate tracings. Am J Obstet Gynecol. 1998 Oct;179(4):1099-100.

2 Schifrin BS, Koos B. Defining the limits of electronic fetal heart rate. Am J Obstet Gynecol. 2017 May;216(5):532.

3 Schifrin BS, Deymier P, Cohen WR. Cranial compression ischemic encephalopathy: fetal neurological injury related to the mechanical forces of labor and delivery. In: Stress and Developmental Programming of Health and Disease: Beyond Phenomenology. Nova Science Publishers, Inc.; 2014.

4 Alfirevic Z, Devane D, Gyte GM, Cuthbert A. Continuous cardiotocography (CTG) as a form of electronic fetal monitoring (EFM) for fetal assessment during labour. Cochrane $\mathrm{Da}$ tabase Syst Rev. 2017 Feb;2:CD006066.

5 Clark SL, Hamilton E, Garite TJ, Timmins A, Warrick PA, Smith S. The Limits of Electronic Fetal Heart Rate Monitoring in the Prevention of Neonatal Metabolic Acidemia. Am J Obstet Gynecol. 2017 Feb;216(2):163.e1-6.

6 Grimes DA, Peipert JF. Electronic fetal monitoring as a public health screening program: the arithmetic of failure. Obstet Gynecol. 2010 Dec;116(6):1397-400.

7 Nelson KB, Dambrosia JM, Ting TY, Grether $\mathrm{JK}$. Uncertain value of electronic fetal monitoring in predicting cerebral palsy. N Engl J Med. 1996 Mar;334(10):613-8.
8 Graham EM, Adami RR, McKenney SL, Jennings JM, Burd I, Witter FR. Diagnostic accuracy of fetal heart rate monitoring in the identification of neonatal encephalopathy. Obstet Gynecol. 2014 Sep;124(3):507-13.

9 Lent M. The medical and legal risks of the electronic fetal monitor. Stanford Law Rev. 1999 Apr;51(4):807-37.

10 Sartwelle TP, Johnston JC. Continuous Electronic Fetal Monitoring during Labor: A Critique and a Reply to Contemporary Proponents. Surg J (NY) 2018 Mar;4(1):e23-e28.

11 Eden RD, Evans MI, Evans SM, Schifrin BS. The "fetal reserve index": re-engineering the interpretation and response to fetal heart rate patterns. Fetal Diagn Ther. 2018;43(2):90104.

12 Eden RD, Evans MI, Evans SM, Schifrin BS. Reengineering Electronic Fetal Monitoring Interpretation: Using the Fetal Reserve Index to Anticipate the Need for Emergent Operative Delivery. Reprod Sci. 2018 Apr;25(4): 487-97.

13 Evans MI, Eden RD, Britt DW, Evans SM, Schifrin BS. Re-engineering the interpretation of electronic fetal monitoring to identify reversible risk for cerebral palsy: a case control series. J Matern Fetal Neonatal Med. 2018 Feb:1-9.
14 Britt DW. A Conceptual Introduction to Modeling. Mahwah (NJ):Lawrence Erlbaum Associates; 1997.

15 Raftery AE. Baysian model selection in social research. In: Marsden PV, editor. Sociological Methodology. London: Tavistoci; 1995. pp. 100-63.

16 Pampel FC. Logistic Regression: A Primer. Thousand Oaks (CA): Sage Publications; 2000.https://doi.org/10.4135/9781412984805.

17 Field A. Discovering Statistics Using IBM SPSS Statistics. 4th ed. Thousand Oaks (CA): Sage Publications; 2012.

18 Britt DW, Eden RD, Evans MI. Matching risk and resources in high-risk pregnancies. J Matern Fetal Neonatal Med. 2006 Oct;19(10): 645-50.

19 Evans MI, Wapner RJ, Berkowitz RL. Noninvasive prenatal screening or advanced diagnostic testing: caveat emptor. Am J Obstet Gynecol. 2016 Sep;215(3):298-305.

20 Evans MI, Evans SM, Bennett TA, Wapner RJ. The price of abandoning diagnostic testing for cell-free fetal DNA screening. Prenat Diagn. 2018 Mar;38(4):243-5.

21 Evans MI, Andriole S, Curtis J, Evans SM, Kessler AA, Rubenstein AF. The epidemic of abnormal copy number variant cases missed because of reliance upon noninvasive prenatal screening. Prenat Diagn. 2018 Sep;38(10): $730-4$.
Improving the Prediction of Emergency Delivery with the Fetal Reserve Index 